\title{
DWT-based blind and robust watermarking using SPIHT algorithm with applications in tele-medicine
}

\author{
TOSHANLAL MEENPAL \\ Department of Electronics and Telecommunication, National Institute of Technology Raipur, Raipur 492010, \\ India \\ e-mail: tmeenpal.etc@nitrr.ac.in
}

MS received 14 June 2016; revised 6 April 2017; accepted 23 May 2017; published online 7 February 2018

\begin{abstract}
Malicious manipulation of digital images cannot be ignored in certain critical application like telemedicine, where medical records of the patients (mostly in the form of diagnostic images of brain, chest, heart, lungs, etc.) need to be securely transmitted over the internet for various life-saving consultation and treatments. Watermarking is used to protect such documents from being manipulated and helps detect the authenticity of the image received. A robust and blind image watermarking technique based on discrete wavelet transform is proposed in this paper. For embedding purpose, suitable coefficients are found out using the Set Partitioning in Hierarchical Trees algorithm. Level of security is further enhanced by scrambling the watermark using Arnold transform and by making the set of embedding locations random using a secret key. Noise Visibility Function is used to compute the minimum allowable distortion for each coefficient before embedding to achieve better image fidelity. Experimental results show that the proposed scheme is robust against certain standard image processing attacks and outperforms the prior existing similar types of watermarking schemes.
\end{abstract}

Keywords. Arnold transform; discrete wavelet transform (DWT); tele-medicine; Noise Visibility Function (NVF); Set Partitioning In Hierarchical Trees (SPIHT).

\section{Introduction}

Use of transmission of medical information among medical institutions has become more popular nowadays. It has been greatly supported by latest technologies in the field of computer communication network. Rapid developments in the field of tele-medicine, tele-diagnosis and tele-consultancy services have saved many human lives. The major component of data being used in this field consists of medical images [1], which helps in instant diagnosis. Several cases have been reported where the crucial diseases have been identified and understood very quickly by the circulation of the medical images through public network instantly to the chain of experienced doctors around the world. Together with these many advantages, there are a few concerns too, for example, easy duplication of the data, manipulation of the original diagnostic images as well as unauthorized distribution of the sensitive medical data [2]. Digital Imaging and Communication in Medicine (DICOM) standards have been proposed for exchange of the medical data through public network. However, they are not secure and reliable enough. There are several cases where header information is manipulated or completely removed [3, 4]. Image integrity, confidentiality, content authenticity and copyright protection are some of the crucial issues that need to be ensured for any data, although the main concern or the scope of this paper is limited to digital images with more importance to the medical images. Digital watermarking is an effective method for copyright protection, copy protection, proof of ownership, etc. [5]. It is the best suited technique for any type of digital images in general.

Embedding of copyright or other information called watermark into an image/video is called digital watermarking [6]. The extracted watermark may be used to prove ownership, or to retrieve some copyright-related information [7]. Watermark extraction algorithms can use the original image to find the watermark in copyright protection application. This is called non-blind watermarking. The original image is generally not accessible in most of the other applications, like copy protection. Such watermarking techniques are referred to as blind watermarking schemes [8]. Any watermarking technique must satisfy certain requirements like perceptual transparency and robustness. Perceptual transparency of the watermark [7] is an essential requirement, i.e., the watermark should not degrade the quality of the host image. Another essential requirement is robustness of the watermark to common signal processing operations and attacks. In digital watermarking domain, it is considered that the algorithms for embedding and extracting the watermark is known to public domain and the security lies in the secret key involved 
during the watermark insertion process. It must be secure enough that an adversary should not be able to detect the presence of the watermark or remove it [8] without having access to correct key. Embedding strength is varied in order to resist from different types of attacks. However, increasing the strength of embedding degrades the quality of the watermarked image [5]. Therefore, a suitable tradeoff between robustness and the imperceptibility of the watermark needs to be maintained.

Watermarking techniques are categorized into spatial and transform domain techniques based on the embedding domain. Pixel values are directly modified to embed a watermark bit in spatial domain methods. Mukherjee et al [9] proposed a robust spatial domain watermarking scheme, in which it has been shown that watermark recovery is possible even after variety of attacks, whereas in the transform domain techniques, transform coefficients are altered to embed a watermark bit [10]. With a few exceptions like [9], in general, spatial domain techniques are simple and weak while the transform domain techniques are complex and robust against different attacks [11]. Some of the existing transform domain methods are discrete cosine transform (DCT) [12-14], discrete Fourier transform (DFT) [15, 16], finite ridgelet transform (FRIT) [17] and discrete wavelet transform (DWT) $[18,19]$. Singular Value Decomposition (SVD)based watermarking process are content-dependent, which eliminates the degradation of image quality caused by image processing [20]. However, SVD is sensitive to noise. As a result, SVD-based watermarking schemes are often fragile (e.g., [21]). Some approaches also send side information to improve robustness (e.g., [22, 23]). Among the transform domain methods, wavelet-based methods are more popular due to their excellent frequency localization properties. DWT is very suitable for identifying areas in the cover image where a watermark can be embedded imperceptibly due to its excellent spatio-frequency properties. Various DWT-based watermarking techniques have been proposed that provide greater robustness [24].

A watermarking scheme in which the watermark embedding strength is estimated by analysing the quality degradation characteristics of the cover image has been proposed in [25]. Better summarization of local region characteristics of an image for watermark embedding by using DWT and SPIHT is presented in [26]. Hsieh et al [27] proposed an approach using the concept of zero-tree from embedded zero-tree wavelet (EZW) coding introduced by Shapiro [28]. More improvement is achieved by the Set Partitioning in Hierarchical Trees (SPIHT) algorithm proposed by Said in [26]. In this method, more zero-trees are efficiently found and represented by separating the tree root from the tree. The SPIHT-based method has therefore become the core technology of the emerging multimedia standards MPEG-4 and JPEG 2000.
In this paper, a DWT-based robust and blind watermarking scheme is proposed, in which required number of significant coefficients are selected from middle frequency band of transformed image by applying SPIHT algorithm. The maximum allowable distortion of selected coefficient is computed using Noise Visibility Function (NVF) [29]. Embedding positions of watermarked image are randomized using a pseudo-random generator and saved as a public key. The proposed approach has following advantages:

1. the SPIHT compression algorithm makes the proposed scheme resistive against large scale compression, cropping and many other image processing attacks,

2. use of NVF results in better imperceptibility and

3. use of private keys further adds to the security of the proposed scheme.

Rest of the paper is organized as follows. Some basic preliminaries are presented in a brief manner in section 2 . The proposed scheme, which consists of an embedding phase and extraction phase, is presented in detail in section 3. In section 4 all the experimental results as well as comparison with some previous existing schemes are shown. Finally, conclusion has been drawn in section 5 .

\section{Preliminaries}

\section{$2.1 D W T$}

DWT is a mathematical tool for hierarchically decomposing an image, $I$. DWT is useful for processing of nonstationary signals. The transform is based on small waves, called wavelets, of varying frequency and limited duration. Wavelet transform provides both frequency and spatial description of an image. The DWT splits the signal into high and low frequency parts while the low frequency part is split again into high and low frequency parts. After the first level of decomposition, there are 4 sub-bands in the DWT transformed image, $I_{D}$ : LL1, LH1, HL1 and HH1. For each successive level of decomposition, the LL sub-band of the previous level is used as the input. To perform second level decomposition, the DWT is applied to LL1 band, which decomposes the LL1 band into the four sub-bands LL2, LH2, HL2, and HH2. To perform third level decomposition, the DWT is applied to LL2 band, which decompose this band into four sub-bands LL3, LH3, HL3, HH3. This results in 10 sub-bands per component. LH1, HL1 and HH1 contain relatively high frequency information (horizontal, vertical and diagonal details) and are thus called frequency bands present in the image tile, whereas LL3 contains an approximation of the original image and is generally called as the lowest frequency band. The three-level DWT decomposition is shown in figure 1. 


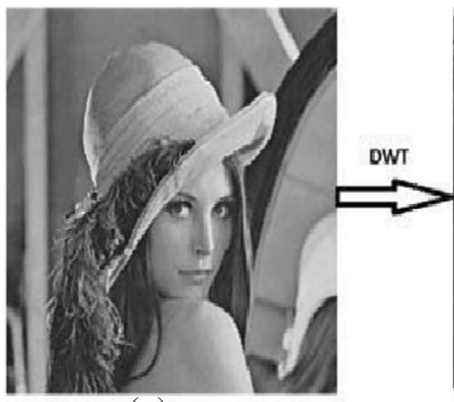

(a)

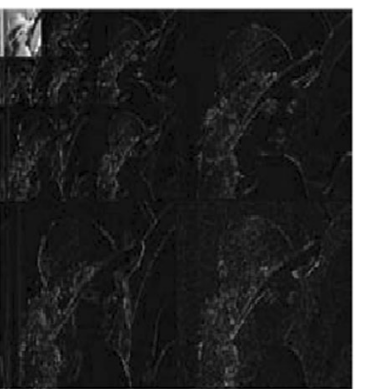

(b)
Figure 1. (a) Test image (Lena) and (b) 3-level 2D DWT decomposition.

\subsection{EZW encoding and SPIHT}

An EZW encoder [28] is an encoder specially designed to be used with wavelet transforms. The EZW encoder was originally designed to operate on images (2D signals) but it can be used also on other dimensional signals. The EZW encoder is based on progressive encoding to compress an image into a bit stream with increasing accuracy. This means that when more bits are added to the stream, the decoded image will contain more details, a property similar to that of JPEG encoded images. Accuracy of encoding can be suitably decided as per the need of the scheme. More details about EZW may be gained from [28].

The SPIHT algorithm [26] is an improvement to the EZW.

\section{$2.3 N V F$}

NVF is the function that characterizes local image properties, identifying texture and edge regions where the watermark should be more strongly embedded [30, 31]. The NVF can be applied in either spatial domain or wavelet domain. Assuming the host image subjects as generalized Gaussian distribution, the NVF, $\phi$, of each wavelet coefficient can be calculated as:

$$
\begin{gathered}
m(i, j)=\frac{1}{R S} \sum_{i+\left\lfloor\frac{R}{2}\right\rfloor}^{i-\left\lfloor\frac{R}{2}\right\rfloor j+\left\lfloor\frac{s}{2}\right\rfloor} \sum_{j+\left\lfloor\frac{S}{2}\right\rfloor}^{i} I(i, j) \\
\sigma^{2}(i, j)=\frac{1}{R S} \sum_{i+\left\lfloor\frac{R}{2}\right\rfloor}^{i-\left\lfloor\frac{R}{2}\right\rfloor} \sum_{j+\left\lfloor\frac{S}{2}\right\rfloor}^{j-\left\lfloor\frac{S}{2}\right\rfloor}[I(i, j)-m(i, j)]^{2} \\
\phi(i, j)=\frac{1}{\left(1+\sigma^{2}(i, j)\right)}
\end{gathered}
$$

where $\sigma^{2}(i, j)$ is the local variance of the image $I$, in a window centred on the coefficient with co-ordinate $(i, j)$. $R \times S$ is the dimension of the window and $m(i, j)$ is the local mean around that window with $I(i, j)$ as the centre of the window. Watson et al [32] presented a quantization matrix (table 1) for perceptually lossless compression. The quantization matrix is composed of the quantization factor of each wavelet level and orientation.

The maximum allowable distortion $(\Delta)$ of a wavelet coefficient at location $(i, j)$ is calculated as

$$
\Delta(i, j)=(1-\phi(i, j)) Q_{\lambda, \theta}+\phi(i, j) s
$$

where quantization factor $Q_{\lambda, \theta}$ is found from quantization matrix, $\lambda, \theta$ are, respectively, the wavelet level and orientation and $s$ is the maximum allowable distortion in the flat region of the image. Here, $\lambda$ is the level that describes the number of $2 \mathrm{D}$ filter stages a component has passed through, and $\theta$ is the orientation to identify the four possible combinations of low pass and high pass filtering the signal has experienced. Generally the index orientations followed are $\{1,2,3,4\}=\{\mathrm{LL}, \mathrm{HL}, \mathrm{HH}, \mathrm{LH}\}$, where low and high are in the order horizontal-vertical. Each combination of level and orientation specifies a single band [32]. Usually $s$ is about 3 . The first term tends to 0 in flat regions since the NVF tends to 1 and the allowable pixel distortion needs to be small, leading to a small value of $s$ [31]. The logic behind this is that, it is expected that the watermark distortions will be visible in flat regions and less visible in textured regions.

\subsection{Arnold transform}

Arnold transform is a $2 \mathrm{D}$ chaotic map for scrambling a digital image. Let us consider the original image of size $N \times N$. Arnold transform is defined as follows:

$$
\left(\begin{array}{l}
i^{\prime} \\
j^{\prime}
\end{array}\right)=\left(\begin{array}{ll}
1 & 1 \\
1 & 2
\end{array}\right)\left(\begin{array}{l}
i \\
j
\end{array}\right) \bmod N
$$

where $i, j \in 0,1,2, \ldots, N 1$. Each pixel $(i, j)$ in the watermark image/logo $\left(I_{W}\right)$ is transformed to another pixel $\left(i^{\prime}, j^{\prime}\right)$ by map (5). When all pixels in the image are transformed, the scrambled image is obtained. Arnold scrambling is a periodic process; hence, the original position of $(i, j)$ coordinates gets back after $F$ iterations. The factor $F$ is called the transform period. Due to the periodicity property, Arnold transform is used in many digital image scrambling processes [33]. Arnold map is the simplest among the various methods for scrambling. This map is area

Table 1. Quantization matrix for 4-level DWT.

\begin{tabular}{lcccc}
\hline & \multicolumn{4}{c}{ Level } \\
\cline { 2 - 5 } Quantization & 1 & 2 & 3 & 4 \\
\hline 1 & 14.049 & 11.106 & 11.363 & 14.500 \\
2 & 23.028 & 14.608 & 12.707 & 14.156 \\
3 & 58.756 & 28.408 & 19.540 & 17.864 \\
4 & 23.028 & 14.685 & 12.707 & 14.156 \\
\hline
\end{tabular}


preserving because the determinant of the transformation matrix is 1 [34]. Furthermore, it can be extended into higher dimensions. This map provides better security due to the increased number of security keys. In order to enhance the security and improve the robustness of the proposed watermarking scheme, Arnold transform is applied on the watermark image $I_{W}$. The scrambled watermark image, $I_{W}^{*}$, can be transmitted over the communication channels without revealing any information to the unauthorized receivers [8]. Another advantage of scrambling is that the error that occurs due to tampering/attack on the watermarked image $I^{*}$ by any adversary in between will not be accumulated in a specific region, but will be scattered throughout the whole watermark image $I_{W}$. This decreases the strength of attack to quite a low extent. To recover $I_{W}$, the scrambling algorithm and the transform period should be known for the extraction process.

\section{Proposed algorithm}

In this section, the proposed algorithm is explained. The proposed method attempts to provide better security, imperceptibility and robustness against the various attacks. This technique is a blind block-based scheme, which embeds the watermark using the Arnold transform. The watermark used for embedding is a binary string, which may be visualized as logo in two-dimensional space. Any other type of watermark image may also be used. The embedding and extraction algorithm is given below.

\subsection{Embedding phase}

During watermark embedding phase, the first step is to perform 3-level 2D DWT on the original/host image (I) to get the transformed image, $I_{D}$. This step results in 10 subbands as shown in figure 1. SPIHT algorithm [26] is performed on LH3 and LH2 sub-bands to find out a set of significant coefficients $(L S P)$. LH3 and $\mathrm{LH} 2$ have been chosen to achieve a better trade-off between robustness and imperceptibility. Now the watermark image $I_{W}$, whose dimension is $P Q$, is scrambled using Arnold transform to generate $I_{W}^{S}$. Suppose $U$ number of scrambling iterations steps were used to generate $I_{W}^{s}$. Store $I_{W}^{s}$ in a one-dimensional array, $W$. Now the highest $4 P Q$ number of coefficients are selected from $L S P$ and stored in an array $Z$. Four coefficients have been taken to embed one single watermark bit in the proposed scheme. One may further increase or decrease the number of coefficients depending on the application requirement. $Z$ is then sorted in descending order with respect to their absolute magnitude values and stored in an array, $Z_{s}$. Corresponding index locations pertaining to $Z$ are also stored simultaneously in an array, $\Pi$. The maximum allowable distortion $\Delta$ is calculated for each coefficient of $Z_{s}$ and accordingly the embedding process is

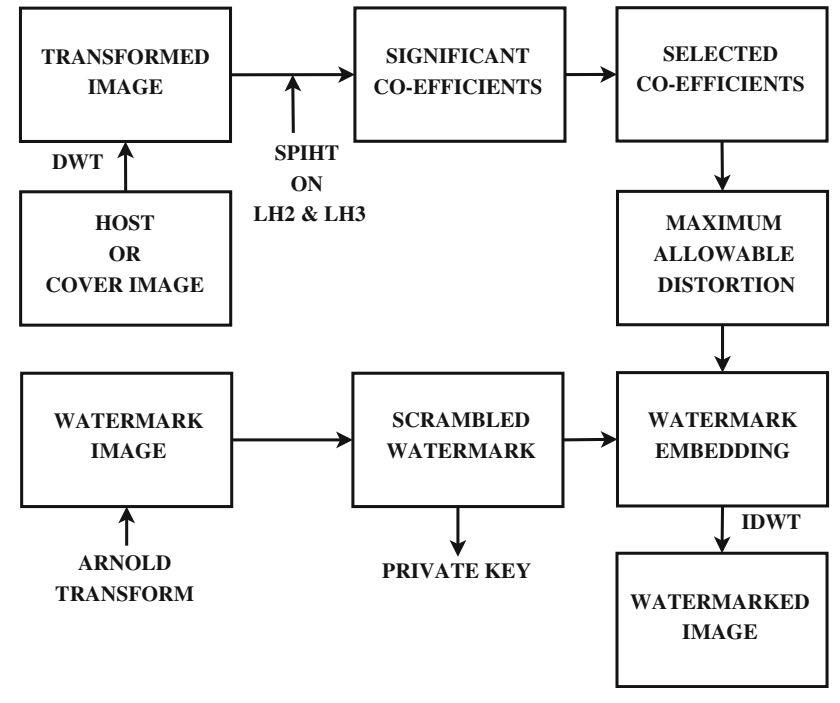

Figure 2. Embedding algorithm.

performed subsequently. Inverse 2D DWT is performed to construct the watermarked image $I^{*}$. Figure 2 shows a block diagram of the proposed embedding algorithm. Various steps involved in the process of embedding are as follows:

1. 3-level DWT is performed on $I$ to decompose it into $I_{D}$, which consists of 10 sub-bands.

2. The SPIHT algorithm is performed on LH3 and LH2 sub-bands to find out significant coefficients, $L S P$.

3. $I_{W}$, whose dimension is $P \times Q$, is scrambled $U$ times using Arnold transform to generate $I_{W}^{s}$.

4. Convert $I_{W}^{S}$ into a one-dimensional array and store it as $W$, where $W$ is a one-dimensional array.

5. The highest $4 P Q$ significant coefficients (according to magnitude) are selected from $L S P$ and stored in $Z$.

6. Sort $Z$ in descending order and store in an array $Z_{s}$.

7. Corresponding index locations pertaining to $Z$ are also stored simultaneously in an array, $\Pi$.

8. $\Delta$ of the selected coefficients are computed.

9. Begin embedding

(a) Select four coefficients at $i, i+1,(i+P Q)$ and $(i+P Q+1)$ positions in vector $Z_{s}$, termed as $X, Y$, $X^{\prime}$ and $Y^{\prime}$, respectively, and $i \in\{1,3,5, \ldots, P Q-3$, $P Q-1\} \cup\{2 P Q+1,2 P Q+3, \ldots, 3 P Q-3,3 P Q-1\}$. Hence, $P Q$ blocks are formed, where each block consists of four coefficients.

(b) Now divide each block into two sub-groups such that $A>B$, where

$$
\begin{aligned}
& A=\operatorname{abs}(X)+\operatorname{abs}\left(X^{\prime}\right) ; \\
& B=\operatorname{abs}(Y)+\operatorname{abs}\left(Y^{\prime}\right) ;
\end{aligned}
$$

Hence, $X$ and $X^{\prime}$ and $Y$ and $Y^{\prime}$ form two sub-groups. 
(c) Now the coefficients are changed by the following rules:

$$
\begin{aligned}
& X_{n e w}=X \pm(1-W(i)) \alpha \Delta ; \\
& X_{n e w}^{\prime}=X^{\prime} \pm(1-W(i)) \alpha \Delta ;
\end{aligned}
$$

where $\alpha$ is the scaling factor. In Eqs. (8) and (9), select positive sign when $X$ or $X^{\prime}$ is negative and vice-versa.

$$
\begin{aligned}
& Y_{\text {new }}=Y \pm(1-W(i)) \alpha \Delta ; \\
& Y_{\text {new }}^{\prime}=Y^{\prime} \pm(1-W(i)) \alpha \Delta ;
\end{aligned}
$$

In Eqs. (10) and (11), select positive sign when $Y$ or $Y^{\prime}$ is positive and negative when $Y$ or $Y^{\prime}$ is negative. This results in

$B-A>$ threshold if $W^{\prime}(i)=0$;

$A>B$ otherwise.

10. Store $X_{\text {new }}, X_{\text {new }}^{\prime}, Y_{\text {new }}$ and $Y_{\text {new }}^{\prime}$ in $Z_{s}^{\prime}$.

11. Place the entries of $Z_{s}^{\prime}$ to their original index locations as initially stored during sorting as $P i$ to form $Z^{\prime}$.

12. Remap the entries of $Z^{\prime}$ to their original positions in $L S P$, which will ultimately result in a 2D 3-level DWTtransformed embedded image, $I_{D}^{E}$.

13. Apply 2D 3-level inverse discrete wavelet transform (IDWT) on $I_{D}^{E}$ to get the watermarked image $I^{*}$.

Embedding positions are required during the extraction process, and, must be sent through some secure channel separately. To add an extra layer of security to the embedding position information, we have applied a randomization process to an array of index locations that contains the embedding positions. Details are given in the next subsection.

\subsection{Creation of private and public key to secure the embedding positions}

We generate a public key $H$ that is an array, using a pseudorandom generator. The pseudo-random generator being used here requires the combination of three private keys. Let us represent these three private keys by $\chi_{1}, \chi_{2}$ and $\chi_{3} . H$ has been considered here as a public key, assuming that, an adversary may get hold of it by successfully attacking the secure channel of transmission. The steps to generate the private key $\left(\chi_{1}, \chi_{2}\right.$ and $\left.\chi_{3}\right)$ and the corresponding public key $H$ is as follows.

1. The seed of pseudo-random number generator is the first component of private key, i.e., $\chi_{1}$.

2. The number of zero bits in watermark image is counted and designated as the second component of private key, i.e., $\chi_{2}$.

3. Suppose the periodicity of $I_{W}$ is $F$. Now, watermark image $I_{W}$ is scrambled $U$ times using Arnold transform

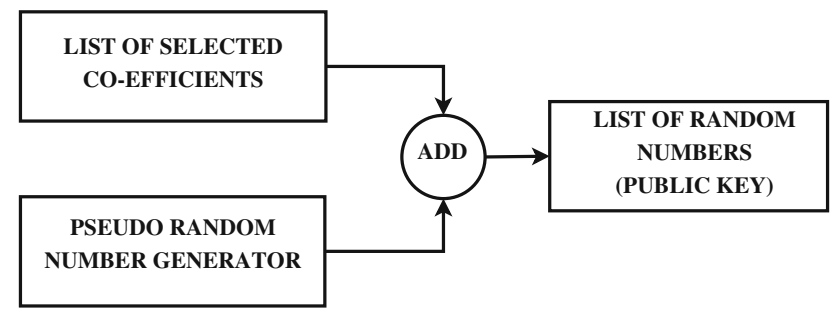

Figure 3. Public key $(H)$ generation.

before the embedding process to generate $I_{W}^{*}$. Then $(F-$ $U)$ will be used as the third component of private key, i.e., $\chi_{3} . F-U$ has been chosen because $F$ may be easily calculated by an adversary although calculation of $F$ is computationally intensive.

4. Generate a pseudo-random sequence $K$ using $\chi_{1}, \chi_{2}$ and $\chi_{3}$.

5. Add $K$ and $\Pi$ to create $H$.

Figure 3 shows a block diagram representation of the public key generation process.

$I^{*}$ may get changed to $I^{\prime}$ due to intentional or un-intentional modification while being transmitted through the public channel. The extraction process to get back the watermark image $I_{W}^{*}$ to verify the authenticity of the received $I^{\prime}$ has been given in the next sub-section.

\subsection{Extraction algorithm}

The extraction process is the inverse of the embedding process. The watermark can be extracted from the distorted watermarked image, $I^{\prime}$, without using $I$ and $I_{W}$. However the set of private keys, i.e., $\chi_{1}, \chi_{2}$ and $\chi_{3}$, and the dimension of $I_{W}$, i.e., $P$ and $Q$, are required for the proper watermark extraction. A block diagram of the watermark extraction algorithm is shown in figure 4. The steps of watermark extraction are as follows:

1. Perform 3-level 2D DWT on the received image $I^{\prime}$.

2. Regenerate $K$ using $\chi_{1}, \chi_{2}$ and $\chi_{3}$ and the same pseudorandom number generator.

3. Subtract $K$ from $H$ to get $\Pi$.

4. Now find image-dependent threshold $\mu$ as follows:

(a) Find blocks where $B>A$ is satisfied.

(b) Compute the difference $\tau=B-A$ in a list $\Omega$.

(c) Select first $K_{p r_{2}}$ entries from $\Omega$.

(d) Compute the threshold as $\mu=\lfloor(\min (\Omega))\rfloor$.

5. Extract watermark bits using the following criterion:

$$
\begin{array}{ll}
W^{\prime}(i)=0 & \text { if } \tau \geq \mu \\
W^{\prime}(i)=1 & \text { otherwise }
\end{array}
$$

where $W^{\prime}(i)$ is scrambled watermark image pixel values in a one-dimensional array. 


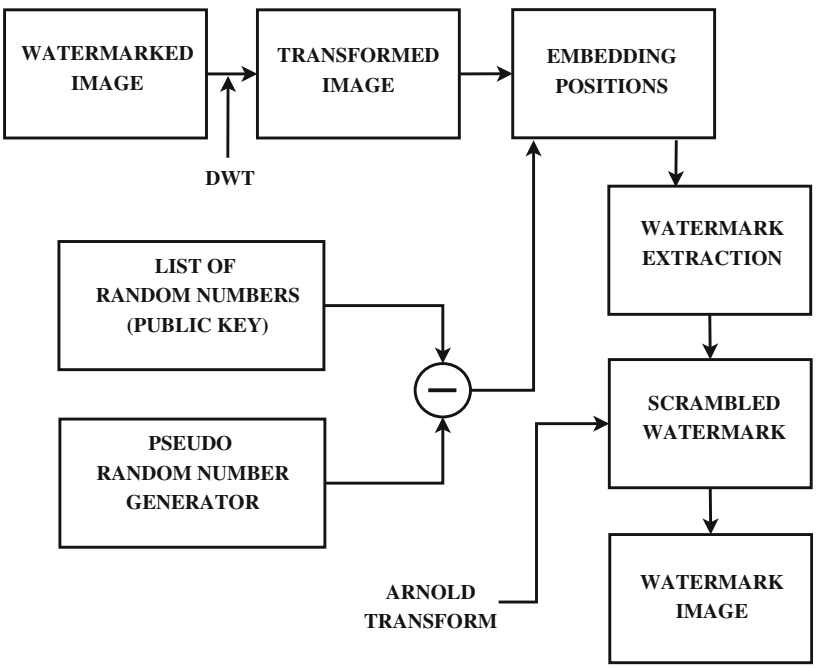

Figure 4. Extraction algorithm.

6. Convert $W^{\prime}(i)$ into $2 \mathrm{D} I_{W}^{s *}$. Here the assumption is that dimension of $I_{W}$ is known to the genuine receiver.

7. Arnold transform is performed on $I_{W}^{* *}$ up to $K_{p r_{3}}$ iterations that yields the extracted watermark image/logo, $I_{W}^{*}$.

\section{Experimental results}

In this section, the performance of the proposed algorithm is tested on standard grey scale images of size $512 \times 512$ pixels, namely Lena, Pirate, Barbara, Baboon and Peppers. The proposed algorithm has also been tested for some medical diagnostic images of spine, heart, lungs, chest and kidney images of a human body. Medical images have been taken randomly from the internet where they were freely available without violating anyone's copyright issues. One may use any watermark image of binary nature for the experiment purpose. The scaling factor $\alpha$ is selected as 3 . Figure 5 shows all the test images being used during the experiment process and watermarks. The watermark image $\left(I_{W}\right)$ is a logo of size $32 \times 32$ as shown in figure 6 . The wavelet used for 2D DWT is Haar wavelet. Value of $U=$ 10 has been chosen for all test images, although it can be varied as per the choice.

\subsection{Imperceptibility analysis}

Peak Signal to Noise Ratio (PSNR) is used for measuring quality of the watermarked image $I^{*}$. PSNR $Q\left(I^{*}, I\right)$ of any image $I^{*}$ with respect to another image $I$ is calculated by the relation

$$
P S N R=10 \log _{10} \frac{M A X_{I}^{2}}{M S E}
$$

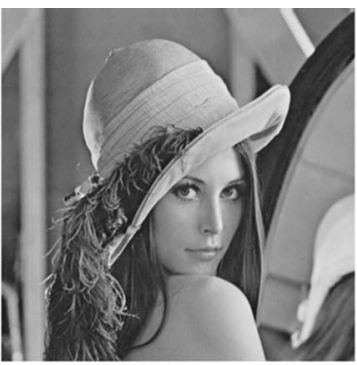

(a) Lena

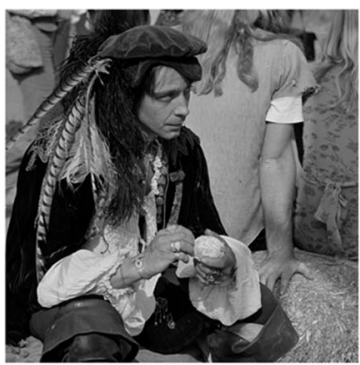

(c) Pirate

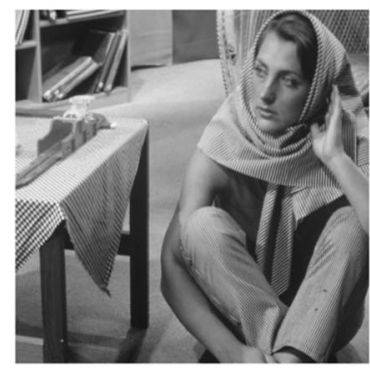

(e) Barbara

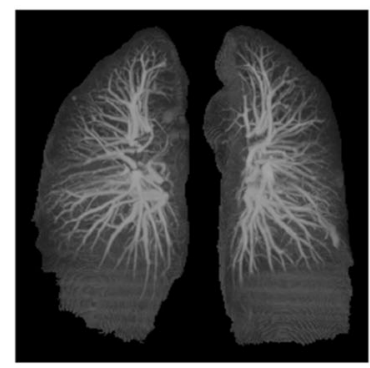

(g) Lung perfusion
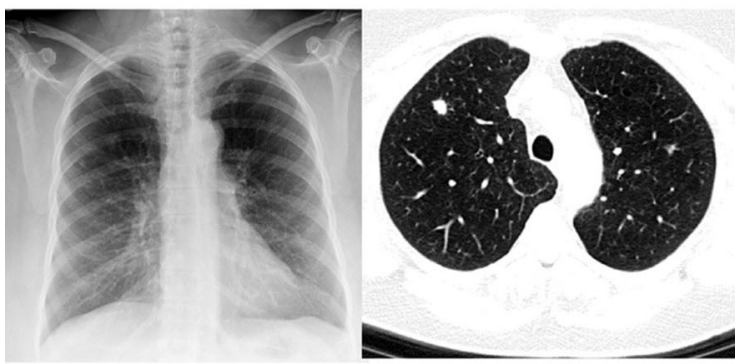

(i) Human chest X-ray and CT scan

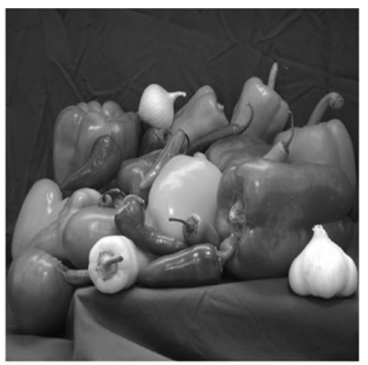

(b) Peppers

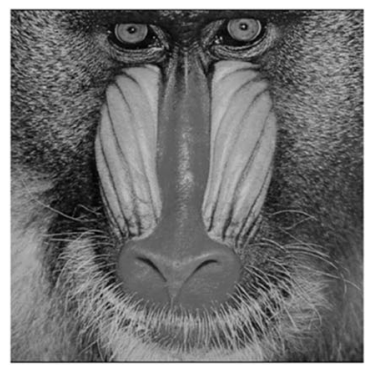

(d) Baboon

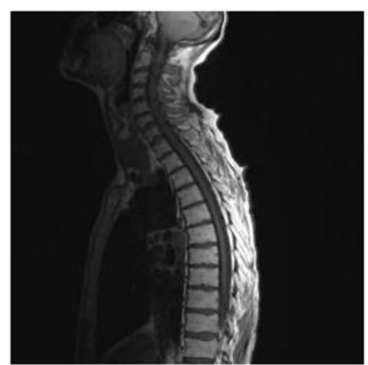

(f) Human spine

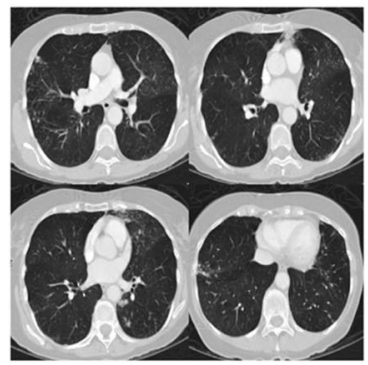

(h) Human lungs
Figure 5. Test images used during experiments. 


\section{ET}

Figure 6. Watermark image/logo.

where $M A X_{I}$ represents the maximum possible pixel value of the image. When the pixels are represented using 8 bits per pixel, this is 255. The $\operatorname{MSE} \phi\left(I^{*}, I\right)$ of any image $I^{*}$ (approximation of $I$ in this case) with respect to another image $I$ is calculated by the relation

$$
M S E=\frac{1}{N M} \sum_{u=1}^{N} \sum_{v=1}^{M}\left[I^{*}(u, v)-I(u, v)\right]^{2}
$$

Figure 7 shows the watermarked images $\left(I^{*}\right)$ for the corresponding test images taken in the previous section. The PSNR of all watermarked images are given in table 2 . From figures 5 and 7, it is quite evident that there is almost no visual difference between the original $(I)$ and watermarked images $\left(I^{*}\right)$.

\subsection{Robustness analysis}

Geometric as well as non-geometric attacks have been applied on the watermarked image followed by the extraction of watermark $W^{\prime}$ from the attacked watermarked image $I^{\prime}$ to analyse the robustness of the proposed algorithm. Various types of attacks like noise addition (salt and pepper noise, Gaussian noise and speckle noise), JPEG compression, filtering process like median filtering, average filtering and Gaussian filtering have been applied. Effects of histogram equalization, cropping and contrast enhancement have also been tested. Normalized correlation, $C$, given by Eq. (14) has been computed to compare the similarity between the original $(W)$ and extracted watermarks $\left(W^{\prime}\right)$. If the proposed scheme is robust enough against certain type of attack then the value of $C$ will remain close to 1 .

$$
C=\frac{\sum(W-\bar{W})\left(W^{\prime}-\bar{W}^{\prime}\right)}{\sqrt{\sum(W-\bar{W})^{2}} \sqrt{\sum\left(W^{\prime}-\bar{W}^{\prime}\right)^{2}}} .
$$

4.2a Effect of noise addition: In this scheme, two types of noise with different variances are added to the watermarked images - salt and pepper noise and speckle noise - to analyse the effect of noise addition. The salt and pepper noise is caused by sharp and sudden disturbances in the image. Its appearance is randomly scattered white or black (or both) pixels over the image. It causes dark pixels in bright regions and bright pixels in dark regions. The result of adding salt and pepper noise to watermarked images is tabulated in table 3. Speckle noise is mainly observed in images obtained from radars. It is a multiplicative noise, i.e., it is in direct proportion to the

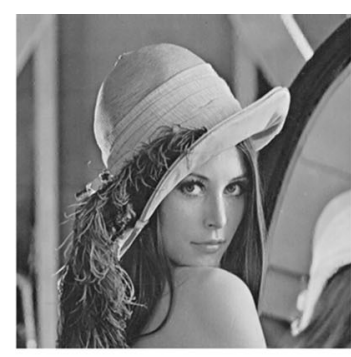

(a) Lena

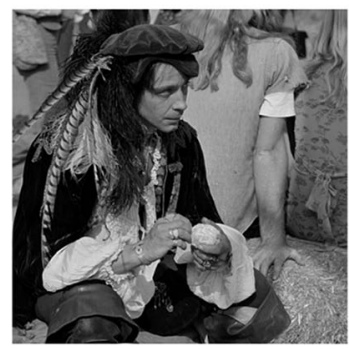

(c) Pirate

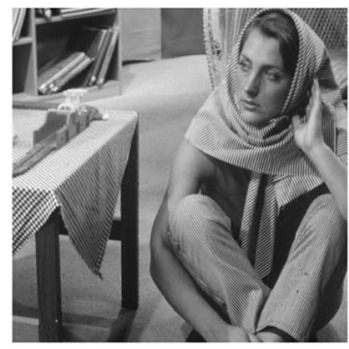

(e) Barbara

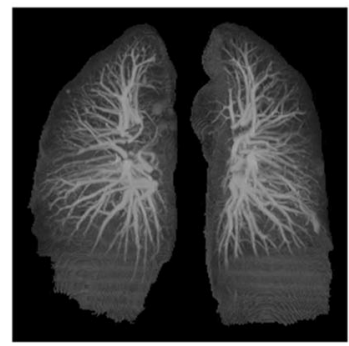

(g) Lung perfusion

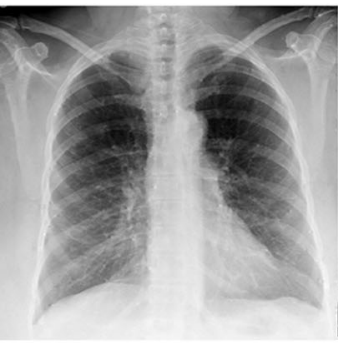

(i) Human chest X-ray and CT scan

Figure 7. Watermarked images generated after embedding process

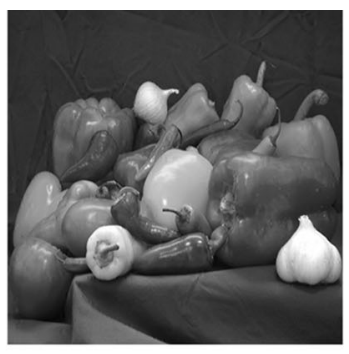

(b) Peppers

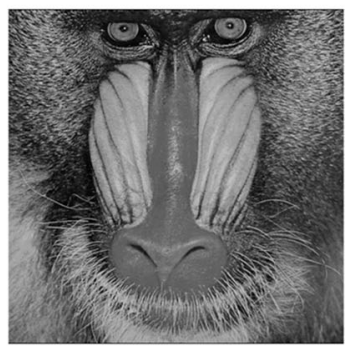

(d) Baboon

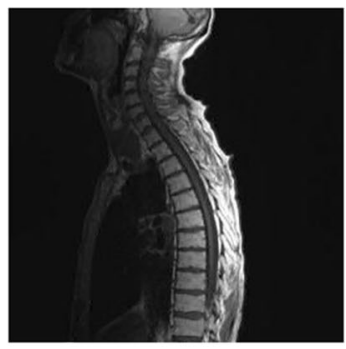

(f) Human spine

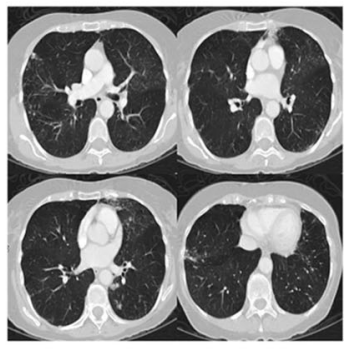

(h) Human lungs

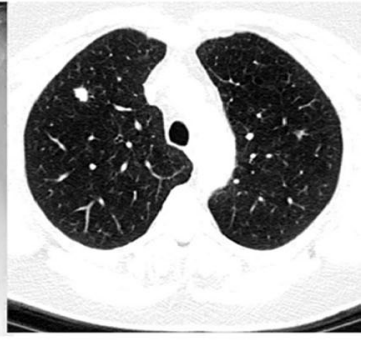


Table 2. PSNR between the original and watermarked images.

\begin{tabular}{lc}
\hline Images & $P S N R(\mathrm{~dB})$ \\
\hline Lena & 46.0999 \\
Peppers & 46.1504 \\
Pirate & 46.4316 \\
Baboon & 46.0817 \\
Barbara & 46.0804 \\
Spine & 46.0613 \\
Lung perfusion & 46.4120 \\
Chest X-ray and CT scan & 46.4616 \\
Lungs & 46.1033 \\
\hline
\end{tabular}

local grey level in any area. It adds multiplicative noise to the image say $I$ such that noisy image $J=(I+n I)$ where $n$ is uniformly distributed random noise. The results of adding speckle noise to watermarked images is tabulated in table 4. Correlation values achieved between the extracted watermarks and original watermark prove the robustness of the algorithm under this kind of attack.

4.2b Effect of filtering: Median filtering, average filtering and Gaussian filtering with the mask size of $3 \times$ 3 are performed on the watermarked image. Normalized correlation values of the extracted watermark with respect to the original watermark are calculated for all test images. The proposed scheme can effectively detect the watermark even when the watermarked image is degraded by various filtering operations, as shown in table 5.

4.2c JPEG compression: Compression is the most common image attacking operation. The watermarked image is compressed with different quality factors ranging from 10 to 70. $C$ values of the extracted watermarks versus the different quality factors are presented in table 6. It may be seen from table 6 that the $C$ values are greater than 0.75 in all the cases, which may be sufficient enough to recognize the watermark.

4.2d Histogram equalization: Histogram equalization provides a sophisticated method for modifying the dynamic range and contrast of an image by altering the image such that its intensity histogram has a desired shape. Results achieved as per table 7 prove the robustness of the proposed scheme against this kind of attack.

4.2e Cropping: A digital image is made up of pixels. Cropping is the process of removing selected pixels from a digital image. It is a lossy operation and considered as one of the most dreadful attacks for image watermarking. Therefore, it has been a big challenge to prevent exploitation of digital data through this attack in the digital world. Table 8 proves that the scheme is quite robust against this kind of attack also.

4.2f Contrast adjustment: Results after applying contrast adjustment of various degrees are given in table 9. Robustness of the scheme is self-evident as per the values of $C$ for different cases enlisted in table 9 .

4.2g StirMark attack: StirMark is a well-known benchmark tool developed for simple robustness testing of image marking algorithms and other steganographic techniques [35]. StirMark attack is basically a resampling process, which involves the combination of certain steps like minor geometric distortion, stretching, shearing, shifting and/ or rotation by some unnoticeable random amount followed by reconstruction using either bi-linear or Nyquist interpolation. In addition, a transfer function is also applied that introduces a small and smoothly distributed error into all sample values. If StirMark is applied once, then a practically unnoticeable quality loss in the image is introduced. Particularly, we are

Table 3. Correlation coefficient $(C)$ for salt and pepper noise attack.

\begin{tabular}{lccccccccc}
\hline & \multicolumn{7}{c}{$C$} \\
\cline { 2 - 8 } Noise density & Lena & Peppers & Pirate & Baboon & Barbara & Spine & Lung perfusion & Chest X-ray and CT scan & Lungs \\
\hline 0.01 & 0.9805 & 0.8638 & 0.9805 & 1 & 0.9564 & 0.8638 & 0.9701 & 0.9832 \\
0.02 & 0.9080 & 0.7549 & 0.9035 & 0.9614 & 0.9319 & 0.8638 & 0.9055 & 0.9102 \\
0.03 & 0.8444 & 0.6587 & 0.8402 & 0.8789 & 0.8991 & 0.8638 & 0.8738 & 0.8964 \\
& & & & & & & 0.8254 \\
\hline
\end{tabular}

Table 4. Correlation coefficient for speckle noise attack.

\begin{tabular}{lcccccccrc}
\hline & \multicolumn{7}{c}{$C$} \\
\cline { 2 - 8 } Noise density & Lena & Peppers & Pirate & Baboon & Barbara & Spine & Lung Perfusion & Chest X-Ray and CT Scan & Lungs \\
\hline 0.02 & 0.9708 & 0.8510 & 0.9854 & 1 & 0.9758 & 0.9531 & 0.9345 & 0.9257 \\
0.03 & 0.9183 & 0.8006 & 0.9416 & 0.9758 & 0.5421 & 0.8109 & 0.9201 & 0.9574 \\
0.04 & 0.8703 & 0.7304 & 0.8886 & 0.9518 & 0.8886 & 0.7402 & 0.8536 & 0.8842 \\
\hline
\end{tabular}


Table 5. Correlation coefficient $(C)$ for filtering attack.

\begin{tabular}{llllllllll}
\hline & \multicolumn{7}{c}{$C$} \\
\cline { 2 - 9 } Filter $(3 \times 3)$ & Lena & Peppers & Pirate & Baboon & Barbara & Spine & Lung perfusion & Chest X-ray and CT scan & Lungs \\
\hline Mean & 0.8550 & 0.9472 & 0.7645 & 0.8222 & 0.4867 & 0.8723 & 0.7754 & 0.8323 \\
Median & 0.9854 & 1 & 0.9319 & 0.9176 & 0.6996 & 0.9532 & 0.9139 & 0.9746 \\
Gaussian & 1 & 1 & 1 & 1 & 1 & 1 & 1 & 1 & 0.7103 \\
\hline
\end{tabular}

Table 6. Correlation coefficient $(C)$ for JPEG compression attack.

\begin{tabular}{lllllllccc}
\hline & \multicolumn{10}{c}{$C$} \\
\cline { 2 - 9 } Quality factor (\%) & Lena & Peppers & Pirate & Baboon & Barbara & Spine & Lung Perfusion & Chest X-Ray and CT Scan & Lungs \\
\hline 70 & 1 & 1 & 1 & 1 & 1 & 1 & 1 & 1 \\
50 & 1 & 0.9902 & 1 & 1 & 1 & 1 & 1 & 1 \\
30 & 1 & 0.9805 & 1 & 1 & 1 & 1 & 0.9975 & 1 \\
20 & 1 & 0.9758 & 1 & 0.9902 & 0.9902 & 0.9876 & 0.9910 & 0.9874 \\
10 & 0.8649 & 0.8141 & 0.8223 & 0.8799 & 0.7877 & 0.8234 & 0.8112 & 0.9872 \\
\hline
\end{tabular}

Table 7. Correlation coefficient $(C)$ for histogram equalization attack.

\begin{tabular}{lcccccccc}
\hline \multicolumn{1}{c}{$C$} & \multicolumn{1}{c}{$c$} \\
\hline Lena & Peppers & Pirate & Baboon & Barbara & Spine & Lung perfusion & Chest X-ray and CT scan & Lungs \\
\hline 0.9708 & 0.4514 & 0.9951 & 0.9902 & 1 & 0.9501 & 0.9945 & 0.9943 \\
\hline
\end{tabular}

Table 8. Correlation coefficient for cropping attack.

\begin{tabular}{|c|c|c|c|c|c|c|c|c|c|}
\hline \multirow[b]{2}{*}{ Cropped area size } & \multicolumn{9}{|c|}{ Correlation coefficient } \\
\hline & Lena & Peppers & Pirate & Baboon & Barbara & Spine & Lung perfusion & Chest X-ray and CT scan & Lungs \\
\hline $64 \times 64$ & 1 & 1 & 0.9854 & 0.9319 & 0.9376 & 0.9987 & 0.9981 & 0.9654 & 0.9456 \\
\hline $128 \times 128$ & 0.9235 & 0.9758 & 0.8541 & 0.8167 & 0.8736 & 0.9586 & 0.9021 & 0.8943 & 0.9021 \\
\hline
\end{tabular}

Table 9. Correlation coefficient $(C)$ for contrast adjustment attack.

\begin{tabular}{lccccccccc}
\hline & \multicolumn{8}{c}{ Correlation coefficient } \\
\cline { 2 - 9 } Contrast increment (\%) & Lena & Peppers & Pirate & Baboon & Barbara & Spine & Lung perfusion & Chest X-ray and CT scan & Lungs \\
\hline 50 & 1 & 0.9951 & 0.9854 & 1 & 1 & 0.9932 & 1 & 0.9763 \\
75 & 1 & 0.9467 & 0.9421 & 1 & 1 & 0.9654 & 0.9342 & 0.9943 \\
100 & 1 & 0.9080 & 0.8222 & 1 & 0.9902 & 0.9108 & $0 . .7890$ & 0.9657 \\
& & & & & & & & & 0.9302 \\
\hline
\end{tabular}

interested in random geometric bending attack as all other attacks have been tested separately and presented here in separate sub-sections. The proposed scheme is not able to survive such attack directly; however, it may be overcome by applying a simple pre-processing technique known as window matching algorithm presented in [36]. Thus, the proposed scheme can overcome this attack also successfully with an added pre-processing step.
Due to space limitations we present the resultant attacked images $\left(I^{\prime}\right)$ and the corresponding watermark images $\left(W^{\prime}\right)$ being extracted out of them for Lena image only in figures 8 and 9. Figure 8 shows the visual appearance of attacked Lena image for various cases of attack mentioned earlier. Figure 9 shows the visual appearance of the corresponding extracted watermark images for each of the cases, whose images are shown in figure 8. 


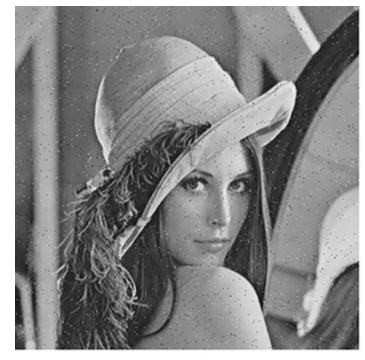

(a)

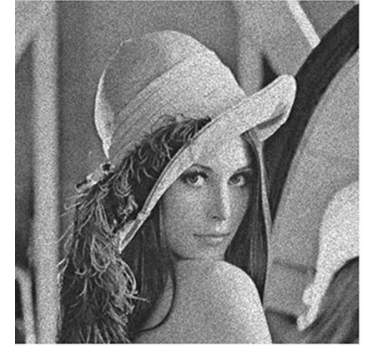

(e)

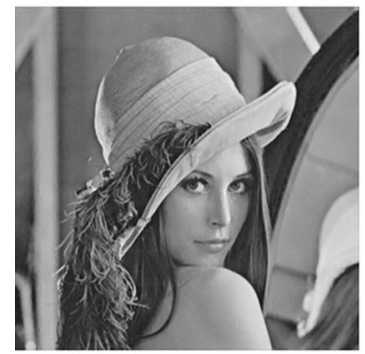

(i)

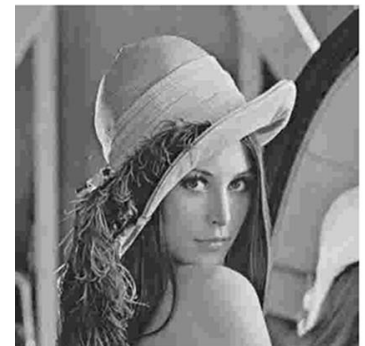

(m)

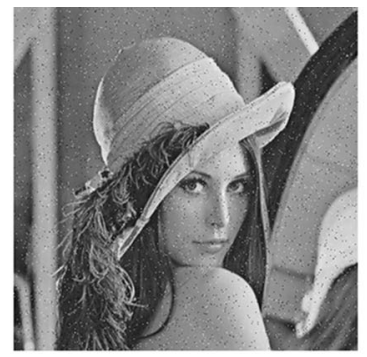

(b)

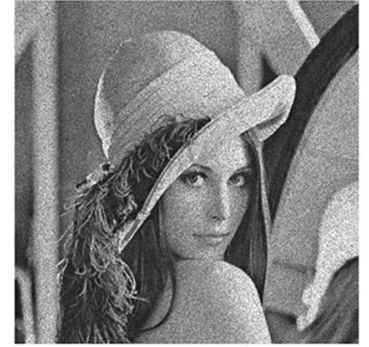

(f)

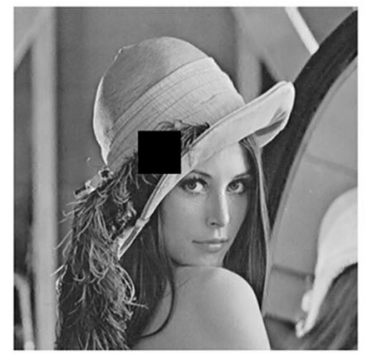

(j)

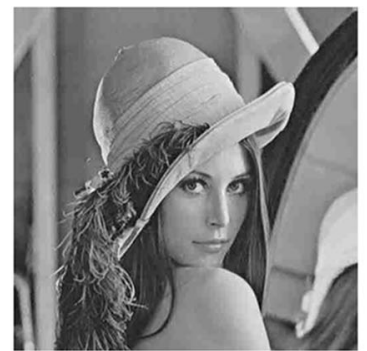

(n)

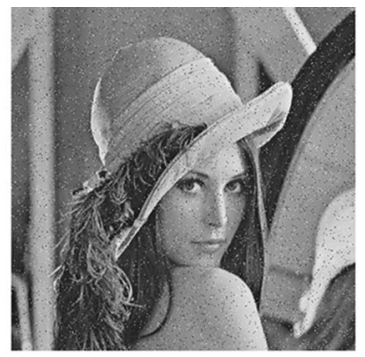

(c)

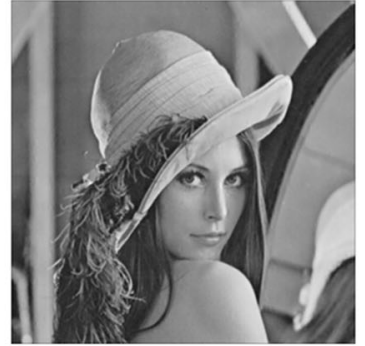

(g)

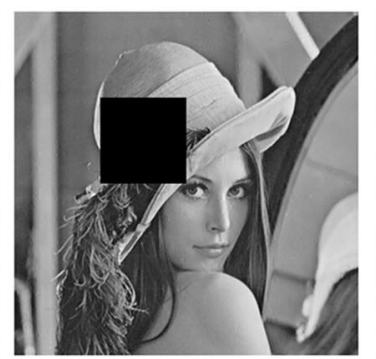

(k)

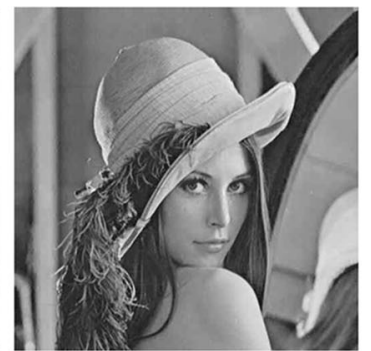

(o)

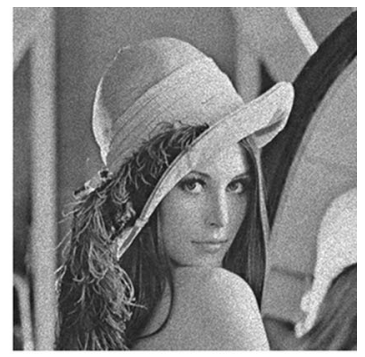

(d)

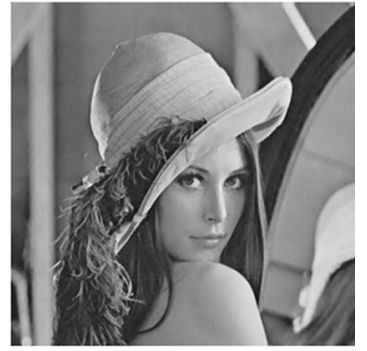

(h)

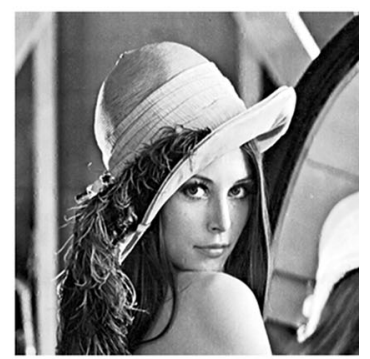

(I)

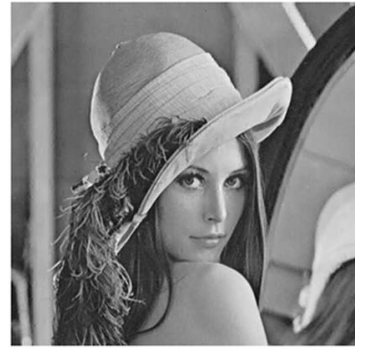

(p)

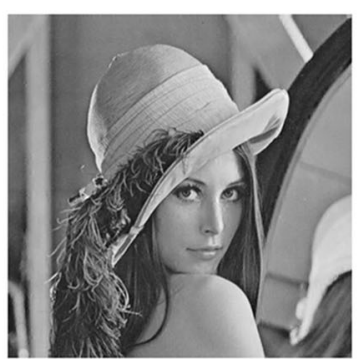

(q) 
4Figure 8. Attacked watermarked images for various cases: salt and pepper noise addition of variance $0.01,0.02$ and 0.03 ; speckle noise addition of variance $0.01,0.02$ and 0.03 ; effect of various filters like average, median and Gaussian of size $3 \times 3$; cropping of size $64 \times 64$ and $128 \times 128$; JPEG compression of quality factors $10,20,30,50$ and 70 .

\section{ET ET ETETET ET}

(a)

\subsection{Comparison to existing methods}

The proposed algorithm is compared to a non-blind scheme presented in [37] and a blind scheme presented in [38]. The comparison results are summarized in tables 10 and 11 . The comparative results are given only for the standard images like Pirate or Lena. Table 10 shows that the proposed method achieves almost similar result or better in some cases even though the scheme proposed in [37] is a non-blind scheme and our proposed scheme is a blind scheme. Comparison of results according to correlation coefficient $(C)$ with the blind scheme proposed in [38] is listed in table 11. In this case the proposed scheme outperforms the scheme proposed in [38] for each type of case.

\section{Conclusion}

A new blind image watermarking scheme in the DWT domain using SPIHT algorithm has been proposed in this paper. To further enhance the security of the scheme, Arnold scrambling is applied on the watermark image. During extraction, only the private key and dimension of the watermark image are required. Although the embedding is performed in the low frequency band, still the imperceptibility criterion is achieved very well by the proposed scheme. SPIHT algorithm is used to select significant coefficients out of the DWT coefficients. The robustness of the scheme is tested by a variety of attacks and the results are compared to those from the existing methods. The watermark can be effectively extracted from the significantly distorted image resultant from various attack operations like JPEG compression, additive noise, median filtering, average filtering, Gaussian filtering, histogram equalization, cropping and contrast manipulation. In total, the proposed scheme has been able to meet three important properties: security, imperceptibility and robustness, and makes a trade-off among them.
Table 11. Robustness comparison (correlation coefficient) with blind scheme for Lena.

\begin{tabular}{clc}
\hline Attack & $\begin{array}{c}\text { Proposed } \\
\text { scheme }\end{array}$ & $\begin{array}{c}\text { Scheme in } \\
{[38]}\end{array}$ \\
\hline JPEG $(Q F=70 \%)$ & 1 & 0.9988 \\
JPEG $(Q F=50 \%)$ & 1 & 0.9952 \\
Gaussian filter $(3 \times 3)$ & 1 & 0.9915 \\
$\quad(m=0, v=0.5)$ & & \\
Median filter $(3 \times 3)$ & 0.9834 & 0.9701 \\
Salt and pepper noise & 0.9224 & 0.9189 \\
$\quad(m=0, v=0.02)$ & & \\
Speckle noise $(m=0, v=0.005)$ & 1 & 0.8547 \\
$\quad$ Gaussian noise & 1 & 0.9368 \\
$(m=0, v=0.001)$ & & \\
\hline
\end{tabular}

\section{References}

[1] Rey Christian and Dugelay Jean-Luc 2002 A survey of watermarking algorithms for image authentication. EURASIP J. Appl. Signal Process. 2002(1): 613-621

[2] Chao Hui-Mei, Hsu Chin-Ming and Miaou Shaou-Gang 2002 A data-hiding technique with authentication, integration, and confidentiality for electronic patient records. Trans. Info. Tech. Biomed. 6(1): 46-53

[3] Ratib Osman and Rosset Antoine 2006 Open-source software in medical imaging: development of osirix. Int. J. Comput. Assist. Radiol. Surgery 1(4): 187-196

[4] Maghraby F, Faheem H M and Roushdy M 2013 A grid based medical image management system using alchemi. Int. J. Comput. Appl. 78(4): 10-16 
[5] Zheng D, Qi H and Zhao J 2007 Human visual system based adaptive digital image watermarking. J. Signal Process. 88: 174-188

[6] Singh Vipula 2011 Digital watermarking: a tutorial. Cyber Journals, Multidisciplinary Journals in Science and Technology, Journal of Selected Areas in Telecommunications (JSAT), January Edition

[7] Liu Jian and He Xiangjian Aug 2005 A review study on digital watermarking. In 2005 International Conference on Information and Communication Technologies, pages 337-341

[8] Langelaar G C, Setyawan I and Lagendijk R L 2000 Watermarking digital image and video data. a state-of-the-art overview. IEEE Signal Process. Mag. 17(5): 20-46

[9] Mukherjee D P, Maitra S and Acton S T 2004 Spatial domain digital watermarking of multimedia objects for buyer authentication. IEEE Trans. Multimedia 6(1): 1-15

[10] Makbol Nasrin M and Khoo Bee Ee 2013 Robust blind image watermarking scheme based on redundant discrete wavelet transform and singular value decomposition. $\{A E U\}$-Int. J. Electron. Commun. 67(2): 102-112

[11] Chen Yongqiang, Zhang Yanqing, Hu Hanping and Ling Hefei 2011 A novel gray image watermarking scheme. J. Softw. 6(5): 849-856

[12] Barni Mauro, Bartolini Franco, Cappellini Vito and Piva Alessandro 1998 A dct-domain system for robust image watermarking. Signal Process. 66(3): 357-372

[13] Huang J, Shi Y Q and Shi Y 2000 Embedding image watermarks in dc components. IEEE Trans. Circuits Syst. Video Technol. 10(6): 974-979

[14] Suhail Mohamed A and Obaidat Mohammad S 2003 Digital watermarking-based dct and jpeg model. IEEE Trans. Instrum. Meas. 52(5): 1640-1647

[15] Ganic Emir and Eskicioglu Ahmet M 2004 A dft-based semiblind multiple watermarking scheme for images. CUNY Brooklyn College 2900

[16] Ganic Emir, Dexter Scott D and Eskicioglu Ahmet M 2005 Embedding multiple watermarks in the dft domain using low- and high-frequency bands

[17] Kalantari N K, Ahadi S M and Vafadust M 2010 A robust image watermarking in the ridgelet domain using universally optimum decoder. IEEE Trans. Circuits Syst. Video Technol. 20(3): 396-406

[18] Sharma Abhilasha, Singh Amit Kumar and Ghrera S P 2015 Secure hybrid robust watermarking technique for medical images. Procedia Comput. Sci. 70: 778-784. Proceedings of the 4th International Conference on Eco-friendly Computing and Communication Systems

[19] Lin Wei-Hung, Wang Yuh-Rau, Horng Shi-Jinn, Kao TzongWann and Pan Yi 2009 A blind watermarking method using maximum wavelet coefficient quantization. Expert Syst. Appl. 36(9): 11509-11516

[20] Andrews H and Patterson C 1976 Singular value decompositions and digital image processing. IEEE Trans. Acoust. Speech Signal Process. 24(1): 26-53

[21] Bao P and Ma Xiaohu 2005 Image adaptive watermarking using wavelet domain singular value decomposition. IEEE Trans. Circuits Syst. Video Technol. 15(1): 96-102
[22] Liu R and Tan T 2002 An svd-based watermarking scheme for protecting rightful ownership. IEEE Trans. Multimedia 4(1): 121-128

[23] Lai C C and Tsai C C 2010 Digital image watermarking using discrete wavelet transform and singular value decomposition. IEEE Trans. Instrum. Meas. 59(11): 3060-3063

[24] Seo Jung-Hee and Park Hung-Bog 2006 Data-hiding method using digital watermark in the public multimedia network. $J$. Inform. Process. Syst. 2(2): 82-87

[25] Wang S, Zheng D, Zhao J, Tam W J and Speranza F 2014 Adaptive watermarking and tree structure based image quality estimation. IEEE Trans. Multimedia 16(2): 311-325

[26] Said Amir and Pearlman William A 1996 A new, fast, and efficient image codec based on set partitioning in hierarchical trees. IEEE Trans. Circuits Syst. Video Technol. 6(3): 243-250

[27] Hsieh M-S, Tseng D-C and Huang Y-H 2001 Hiding digital watermarks using multiresolution wavelet transform. IEEE Trans. Ind. Electron. 48(5): 875-882

[28] Shapiro Jerome M 1993 Embedded image coding using zerotrees of wavelet coefficients. IEEE Trans. Signal Process. 41(12): 3445-3462

[29] Zhang Fan, Zhang Xinhong and Zhang Hongbin 2007 Digital image watermarking capacity and detection error rate. Pattern Recogn. Lett. 28(1): 1-10

[30] Voloshynovskiy Sviatoslav, Pereira Shelby, Iquise Victor and Pun Thierry 2001 Attack modelling: towards a second generation watermarking benchmark. Signal Process. 81(6): 1177-1214

[31] Pereira Shelby, Voloshynoskiy S and Pun Thierry 2001 Optimal transform domain watermark embedding via linear programming. Image Process. 81(6): 1251-1260

[32] Watson Andrew B, Yang Gloria Y, Solomon Joshua A and Villasenor John 1997 Visibility of wavelet quantization noise. IEEE Trans. Image Process. 6(8): 1164-1175

[33] Mehta Rajesh, Rajpal Navin and Vishwakarma Virendra P 2015 Robust image watermarking scheme in lifting wavelet domain using ga-lsvr hybridization. Int. J. Mach. Learn. Cybern. pp. 1-17

[34] Ghebleh Mohammad and Kanso Ali 2014 A blind chaosbased watermarking technique. Security Commun. Netw. 7(4): 800-811

[35] Petitcolas Fabien A P, Anderson Ross J and Kuhn Markus G 1998 Attacks on Copyright Marking Systems, pages 218-238. Springer Berlin Heidelberg, Berlin, Heidelberg

[36] Das Tanmoy Kanti and Maitra Subhamoy 2002 A robust block oriented watermarking scheme in spatial domain, pages 184-196. Springer Berlin Heidelberg, Berlin, Heidelberg

[37] Shahid A R Bin 2012 An image watermarking approach based on set partitioning in hierarchical trees (spiht) algorithm. In 2012 International Conference on Informatics, Electronics \& Vision (ICIEV), pages 487-492. IEEE

[38] Shijie R and Xin S 2009 Digital watermarking based on fast independent component analysis and discrete wavelet transform. In 2009 International Conference on Computational Intelligence and Security, vol. 2, pages 341-343 